\title{
Research Article \\ Fixed Point Results for Multivalued Maps in Metric Spaces with Generalized Inwardness Conditions
}

\author{
M. Frigon \\ Département de Mathématiques et de Statistique, Université de Montréal, C.P. 6128, succ. Centre-Ville, \\ Montréal, (QC), Canada H3C 3J7 \\ Correspondence should be addressed to M. Frigon, frigon@dms.umontreal.ca \\ Received 19 November 2009; Accepted 12 February 2010 \\ Academic Editor: Tomonari Suzuki \\ Copyright (C) 2010 M. Frigon. This is an open access article distributed under the Creative \\ Commons Attribution License, which permits unrestricted use, distribution, and reproduction in \\ any medium, provided the original work is properly cited. \\ We establish fixed point theorems for multivalued mappings defined on a closed subset of a \\ complete metric space. We generalize Lim's result on weakly inward contractions in a Banach \\ space. We also generalize recent results of Azé and Corvellec, Maciejewski, and Uderzo for \\ contractions and directional contractions. Finally, we present local fixed point theorems and \\ continuation principles for generalized inward contractions.
}

\section{Introduction and Preliminaries}

In the following $X$ denotes a complete metric space. The open ball centered in $x \in X$ of radius $r>0$ is denoted $B(x, r)$. For $A, B$ two nonempty, closed subsets of $X$, the generalized Hausdorff metric is defined by

$$
D(A, B)=\max \left\{\sup _{a \in A} d(a, B), \sup _{b \in B} d(b, A)\right\} .
$$

Definition 1.1. Let $K \subset X$; we say that the multivalued map $F: K \rightarrow X$ is a contraction if $F$ has nonempty, closed values, and there exists $k \in[0,1[$ such that

$$
D(F(x), F(y)) \leq k d(x, y) \quad \forall x, y \in X \text {. }
$$

The constant $k$ is called the constant of contraction. 
The well known Nadler fixed point Theorem [1] says that a multivalued contraction on $X$ to itself has a fixed point. However, to insure the existence of a fixed point to a multivalued contraction defined on a closed subset $K$ of $X$, extra assumptions are needed.

In 2000, Lim [2] obtained the following fixed point theorem for weakly inward multivalued contractions in Banach spaces using the transfinite induction.

Theorem 1.2. Let $K$ be a nonempty closed subset of a Banach space $E$ and $F: K \rightarrow E$ a multivalued contraction with closed values. Assume that $F$ is weakly inward, that is,

$$
F(x) \subset \overline{\{x+h(u-x): u \in K, h \geq 1\}} .
$$

Then F has a fixed point.

Observe that in the definition of weakly inward maps, linear intervals play a crucial role. Indeed, $y=x+h(u-x)$ for some $u \in K \backslash\{x\}$ and $h \geq 1$ if and only if

$$
u \in\{(1-t) x+t y: 0<t \leq 1\} \cap K
$$

Moreover, $\|x-u\|+\|u-y\|=\|x-y\|$.

From this observation, generalizations of this result to complete metric spaces were recently obtained with simpler proofs by Azé and Corvellec [3], and by Maciejewski [4]. They generalized the inwardness condition using the metric left-open segment

$$
] x, y]=\{z \in X \backslash\{x\}: d(x, z)+d(z, y)=d(x, y)\}
$$

which should be nonempty for every $y \in F(x) \backslash\{x\}$ and "close enough" of $K$. They also obtained results for directional $k$-contractions in the sense of Song [5]. In 2005, Uderzo [6] established a local fixed point theorem for directional $k(\cdot)$-contractions.

In this paper, we generalize their results. More precisely, we first generalize the inwardness conditions used in [2-4]. In particular, for $y \in F(x) \backslash\{x\}$ with $y \notin K$, one can have $] x, y]=\{y\}$. Also, we slightly generalize the notion of $k$-directional contractions.

Finally, we present local fixed point theorems and continuation principles generalizing results of Maciejewski [4] and Uderzo [6]. following.

Here is the well known Caristi Theorem [7] which will play a crucial role in the

Theorem 1.3 (Caristi [7]). Let $f: X \rightarrow X$ and a map $\phi: X \rightarrow \mathbb{R}$ lower semicontinuous and bounded from below such that

$$
d(x, f(x)) \leq \phi(x)-\phi(f(x)) \quad \forall x \in X
$$

Then $f$ has a fixed point.

This result, which is equivalent to the Ekeland variational Principle [8, 9], can also be deduced from the Bishop-Phelps theorem. The following formulation appeared in [10] (see also [11]) while the original formulation appeared in a different form in [12] (see also [13]). 
Theorem 1.4 ( Theorem (Bishop and Phelps)). Let $\phi: X \rightarrow \mathbb{R}$ be lower semicontinuous and bounded from below, and $\lambda>0$. Then for any $x_{0} \in X$, there exists $x^{*} \in X$ such that

(i) $\phi\left(x^{*}\right)+\lambda d\left(x_{0}, x^{*}\right) \leq \phi\left(x_{0}\right)$;

(ii) $\phi\left(x^{*}\right)<\phi(x)+\lambda d\left(x, x^{*}\right)$ for every $x \neq x^{*}$.

The interested reader can find a multivalued version of Caristi's fixed point theorem in an article of Mizoguchi and Takahashi [14].

\section{Generalizations of Inward Contractions}

In this section, we obtain fixed point results for contractions defined on a closed subset of a metric space satisfying a generalized inwardness condition.

Theorem 2.1. Let $K$ be a closed subset of $X$, and let $F: K \rightarrow X$ be a multivalued contraction with constant $k \in[0,1[$. Assume that there exits $\theta \in] k, 1]$ such that for every $x \in K$,

$$
F(x) \subset\{x\} \cup\{y \in X: \exists u \in K \backslash\{x\} \text { such that } \theta d(x, u)+d(u, y) \leq d(x, y)\}
$$

Then F has a fixed point.

Proof. Assume that $F$ has no fixed point. Choose $\varepsilon>0$ such that $k(1+2 \varepsilon)<\theta$. Consider on graph $F=\{(x, y) \in K \times X: y \in F(x)\}$ the metric

$$
\widehat{d}\left(\left(x_{1}, y_{1}\right),\left(x_{2}, y_{2}\right)\right)=\frac{\theta}{1+2 \varepsilon} d\left(x_{1}, x_{2}\right)+d\left(y_{1}, y_{2}\right)
$$

Since $F$ is a contraction with closed values, $($ graph $F, \widehat{d})$ is a complete metric space.

Let $(x, y) \in \operatorname{graph} F$. By assumption, there exists $\bar{x} \in K \backslash\{x\}$ such that

$$
\theta d(x, \bar{x})+d(\bar{x}, y) \leq d(x, y)
$$

Since $y \in F(x)$ and

$$
D(F(x), F(\bar{x})) \leq k d(x, \bar{x})<\frac{\theta}{1+2 \varepsilon} d(x, \bar{x}),
$$

there exists $\bar{y} \in F(\bar{x})$ such that

$$
d(y, \bar{y})<\frac{\theta}{1+2 \varepsilon} d(x, \bar{x})
$$


Therefore,

$$
\begin{aligned}
\varepsilon \widehat{d}((x, y),(\bar{x}, \bar{y}))+d(\bar{x}, \bar{y}) & \leq \frac{\varepsilon \theta}{1+2 \varepsilon} d(x, \bar{x})+\varepsilon d(y, \bar{y})+d(\bar{x}, y)+d(y, \bar{y}) \\
& \leq \theta d(x, \bar{x})+d(\bar{x}, y) \\
& \leq d(x, y) .
\end{aligned}
$$

Defining $f:$ graph $F \rightarrow$ graph $F$ and $\phi:$ graph $F \rightarrow \mathbb{R}$, respectively, by

$$
f(x, y)=(\bar{x}, \bar{y}), \quad \phi(x, y)=\frac{d(x, y)}{\varepsilon}
$$

we deduce from Caristi's theorem (Theorem 1.3) that $f$ has a fixed point which is a contradiction since $x \neq \bar{x}$. So, $F$ has a fixed point.

As a corollary, we obtain Maciejewski's result [4] which generalizes Lim's fixed point theorem for weakly inward multivalued contractions in Banach spaces [2].

Corollary 2.2 (Maciejewski [4]). Let $K$ be a closed subset of $X$, and let $F: K \rightarrow X$ be a multivalued contraction such that for every $x \in K$,

$$
F(x) \subset \tilde{I}_{K}(x)=\{x\} \cup\left\{y \in X \backslash\{x\}: \inf _{z \in] x, y]} \frac{d(z, K)}{d(z, x)}=0\right\}
$$

Then F has a fixed point.

Proof. Let $k \in[0,1$ [ be a constant of contraction of $F$. Fix $\delta \in] 0,(1-k) /(1+k)$ [. One can choose $\theta \in] k,(1-\delta) /(1+\delta)[$. If $y \in F(x) \backslash\{x\}$, there exists $z \in] x, y]$ such that

$$
\frac{d(z, K)}{d(z, x)}<\delta
$$

Thus, there exists $u \in K$ such that $d(z, u)<\delta d(z, x)$. So

$$
\begin{aligned}
\theta d(x, u)+d(u, y) & \leq \theta d(x, z)+(\theta+1) d(z, u)+d(z, y) \\
& \leq \theta d(x, z)+(\theta+1) \delta d(x, z)+d(z, y) \\
& \leq d(x, z)+d(z, y) \\
& =d(x, y)
\end{aligned}
$$

Thus $F(x)$ satisfies (2.1).

From the proof of Theorem 2.1, one sees that one can weaken the assumption that $F$ is a contraction, and hence one can generalize a result due to Azé and Corvellec [3]. 
Theorem 2.3. Let $K$ be a closed subset of $X$ and let $F: K \rightarrow X$ be a multivalued map with nonempty values and closed graph. Assume that there are constants $k \in[0,1[$ and $\theta \in] k, 1]$ such that for every $x \in K$,

$$
\begin{aligned}
& F(x) \subset\{x\} \cup\{y \in X: \exists u \in K \backslash\{x\} \\
& \text { such that } d(y, F(u)) \leq k d(x, u) \leq \theta d(x, u)+d(u, y) \leq d(x, y)\} .
\end{aligned}
$$

Then F has a fixed point.

Corollary 2.4 (Azé and Corvellec [3]). Let $K$ be a closed subset of $X$, and let $F: K \rightarrow X$ be a multivalued map with nonempty values and closed graph. Assume that there exists $k \in[0,1[$ and $\delta>0$ such that $k<(1-\delta) /(1+\delta)$ and for every $x \in K$ and every $y \in F(x) \backslash\{x\}$ there exist $z \in] x, y]$ and $u \in K$ such that

$$
d(u, z)<\delta d(x, z), \quad d(y, F(u)) \leq k d(x, u)
$$

Then F has a fixed point.

Proof. Choose $\theta \in] k,(1-\delta) /(1+\delta)[$. It is easy to see that $F(x)$ satisfies $(2.11)$ for every $x \in K$.

Remark 2.5. Observe that in Theorems 2.1 and 2.3, one can have for some $y \in F(x) \backslash\{x\}$,

$$
\{y\}=] x, y], \quad \frac{d(y, K)}{d(x, y)} \geq \frac{1-k}{1+k}
$$

So

$$
\inf _{z \in] x, y]} \frac{d(z, K)}{d(x, y)} \neq 0, \quad d(u, y)>\delta d(x, y) \quad \forall u \in K, \forall \delta>0 \text { such that } k<\frac{1-\delta}{1+\delta}
$$

Therefore, (2.8) and (2.12) are not satisfied.

Example 2.6. Let $X=\left\{(a, b) \in \mathbb{R}^{2}: a b=0\right\}, K=[(0,0),(1,0)], F: K \rightarrow X$ defined by $F(t, 0)=$ $[(0,0),(0, t / 2)] . F$ is a contraction with constant $k=1 / 2$. Take $x=(1,0)$ and $y=(0,1 / 2) \in$ $F(x)$. Observe that $] x, y]=\{y\}$, and $d(y, K)=1 / 2 \geq \sqrt{5} / 6=d(x, y)(1-k) /(1+k)$. So, $(2.8)$ and (2.12) are not satisfied. On the other hand, choose $\theta=(\sqrt{5}-1) / 2$. Let $x=(t, 0) \in K$ with $t \in] 0,1]$. For all $y \in F(x)$, there exists $s \in[0,1]$ such that $y=(0, s t / 2)$. So, taking $u=(0,0)$, one has

$$
\theta d(x, u)+d(u, y)=\frac{\sqrt{5}-1}{2} t+\frac{s t}{2} \leq \frac{t}{2} \sqrt{4+s^{2}}=d(x, y)
$$

Hence $F(x)$ satisfies all the assumptions of Theorem 2.1 and in particular condition (2.1). 
In the previous results, $F$ is a contraction or has to satisfy a type of contractive condition in some direction, namely,

$$
\forall y \in F(x) \backslash\{x\}, \quad \exists u \in K \backslash\{x\} \text { such that } d(y, F(u)) \leq k d(x, u) \text {. }
$$

A careful look at their proofs permits to realize that a wider class of maps can be considered. Indeed, it is easy to see that the previous results are corollaries of the following theorem which is a direct consequence of Theorem 1.3.

Theorem 2.7. Let $K$ be a closed subset of $X$ and let $F: K \rightarrow X$ be a multivalued map with nonempty values and closed graph. Assume that there exists $\tilde{d}$ an equivalent metric on graph $F$ such that for every $x \in K$ and every $y \in F(x) \backslash\{x\}$,

$$
\exists u \in K \backslash\{x\}, \quad \exists v \in F(u) \text { such that } \tilde{d}((x, y),(u, v))+d(u, v) \leq d(x, y) .
$$

Then F has a fixed point.

Corollary 2.8. Let $K$ be a closed subset of $X$ and let $F: K \rightarrow X$ be a multivalued map with nonempty values and closed graph. Assume that there exists $\alpha, \beta>0$ such that for every $x \in K$,

$$
\begin{array}{r}
F(x) \subset\{x\} \cup\{y \in X: \exists u \in K \backslash\{x\}, \exists v \in F(u) \\
\text { such that } \alpha d(x, u)+d(u, v)+\beta d(v, y) \leq d(x, y)\} .
\end{array}
$$

Then F has a fixed point.

Corollary 2.9. Let $K$ be a closed subset of $X$, and let $f: K \rightarrow X$ be a continuous map. Assume that there exists $\alpha, \beta>0$ such that for every $x \neq f(x)$, there exists $u \in K \backslash\{x\}$ such that

$$
\alpha d(x, u)+d(u, f(u))+\beta d(f(u), f(x)) \leq d(x, f(x)) .
$$

Then $f$ has a fixed point.

Example 2.10. Let $f:[0,1] \rightarrow \mathbb{R}$ be defined by $f(x)=-2 x$. Obviously, $f$ is expansive and satisfies the assumptions of the previous corollary. It does not satisfies (2.1) and (2.11).

\section{Intersection Conditions}

Observe that even though Theorem 2.7 generalizes Theorems 2.1 and 2.3, Condition (2.17) is quite restrictive in the multivalued context since every $y \in F(x) \backslash\{x\}$ has to satify a suitable condition. Here is a fixed point result where at least one element of $F(x)$ has to be in a suitable set.

Theorem 3.1. Let $K$ be a closed subset of $X$, and let $F: K \rightarrow X$ be a multivalued contraction with constant $k \in[0,1[$. Assume that there exits $\theta \in] k, 1]$ such that for every $x \in K$,

$$
\emptyset \neq F(x) \cap(\{x\} \cup\{y \in X: \exists u \in K \backslash\{x\} \text { such that } \theta d(x, u)+d(u, y) \leq d(x, F(x))\}) .
$$

Then F has a fixed point. 
Proof. Assume that $F$ has no fixed point. Let $x \in K$. By assumption, there exist $y \in F(x)$ and $\bar{x} \in K \backslash\{x\}$ such that

$$
\theta d(x, \bar{x})+d(\bar{x}, y) \leq d(x, F(x))
$$

Therefore,

$$
\begin{aligned}
(\theta-k) d(x, \bar{x})+d(\bar{x}, F(\bar{x})) & \leq(\theta-k) d(x, \bar{x})+d(\bar{x}, y)+d(y, F(\bar{x})) \\
& \leq(\theta-k) d(x, \bar{x})+d(\bar{x}, y)+D(F(x), F(\bar{x})) \\
& \leq \theta d(x, \bar{x})+d(\bar{x}, y) \\
& \leq d(x, F(x)) .
\end{aligned}
$$

Defining $f: K \rightarrow K$ and $\phi: K \rightarrow \mathbb{R}$, respectively, by

$$
f(x)=\bar{x}, \quad \phi(x)=\frac{d(x, F(x))}{\theta-k}
$$

we deduce from Caristi's Theorem (Theorem 1.3) that $f$ has a fixed point which is a contradiction since $x \neq \bar{x}$. So, $F$ has a fixed point.

Example 3.2. Let $X=\left\{(a, b) \in \mathbb{R}^{2}: a b=0\right\}, K=[(0,0),(1,0)], F: K \rightarrow X$ defined by $F(t, 0)=\{(t-1) / 2,0),(0, t / 2)\}$. Observe that $(2.1)$ is not satisfied. Indeed, for $x=(0,0)$ and $y=(-1 / 2,0)$, we have $y \in F(x) \backslash\{x\}$ and $d(u, y)>d(x, y)$ for every $u \in K \backslash\{x\}$.

Choose $\theta=(\sqrt{5}-1) / 2$. Let $x=(t, 0) \in K$ with $t \in] 0,1]$, then one has

$$
d(x, F(x))= \begin{cases}\frac{t \sqrt{5}}{2}, & \text { if } t \leq \frac{1}{\sqrt{5}-1} \\ \frac{1+t}{2}, & \text { otherwise. }\end{cases}
$$

Choose $y=(0, t / 2)$ if $t \leq 1 /(\sqrt{5}-1)$, and $y=((t-1) / 2,0)$ otherwise. So, taking $u=(0,0)$, one has

$$
\begin{aligned}
\theta d(x, u)+d(u, y) & = \begin{cases}\left(\theta+\frac{1}{2}\right) t, & \text { if } t \leq \frac{1}{\sqrt{5}-1} \\
\theta t+\frac{1-t}{2}, & \text { otherwise }\end{cases} \\
& \leq d(x, F(x)) .
\end{aligned}
$$

Thus $F$ satisfies all assumptions of Theorem 3.1, and in particular it satisfies (3.1) but does not satisfy (2.1). 
Corollary 3.3. Let $K$ be a closed subset of $X$, and let $F: K \rightarrow X$ be a multivalued contraction with constant $k \in[0,1[$. Assume that there exits $\theta \in] k, 1]$ such that for every $x \in K$,

$$
\begin{aligned}
& \emptyset \neq\{y \in F(x): d(x, y)=d(x, F(x))\} \\
& \quad \cap(\{x\} \cup\{y \in X: \exists u \in K \backslash\{x\} \text { such that } \theta d(x, u)+d(u, y) \leq d(x, y)\}) .
\end{aligned}
$$

Then F has a fixed point.

The previous theorem generalizes a result of Downing and Kirk [15].

Corollary 3.4 (Downing and Kirk [15]). Let $K$ be a closed subset of a Banach space E and F : $K \rightarrow$ $E$ a multivalued contraction such that for every $x \in K$,

$$
\emptyset \neq\{y \in F(x):\|x-y\|=d(x, F(x))\} \cap \overline{\{y=x+h(u-x): u \in K, h \geq 1\}} .
$$

\section{Then F has a fixed point.}

Proof. Let $k \in[0,1[$ be a constant of contraction of $F$. Fix $\theta \in] k, 1[$. For $x \in K$ such that $d(x, F(x)) \neq 0$, there exists $y \in F(x)$ such that $\|x-y\|=d(x, F(x))$ and there exist sequences $\left\{h_{n}\right\}$ in $\left[1, \infty\left[\right.\right.$ and $\left\{u_{n}\right\}$ in $K$ such that $x+h_{n}\left(u_{n}-x\right) \rightarrow y$. Choose $\bar{n}$ big enough such that $\left\|x+h_{\bar{n}}\left(u_{\bar{n}}-x\right)-y\right\|<(1-\theta)\|x-y\| /(1+\theta)$. So $u_{\bar{n}} \in K \backslash\{x\}$ and

$$
\begin{aligned}
\theta\left\|x-u_{\bar{n}}\right\|+\left\|u_{\bar{n}}-y\right\| \leq & \frac{\theta}{h_{\bar{n}}}\|x-y\|+(\theta+1)\left\|u_{\bar{n}}-\left(x+\frac{1}{h_{\bar{n}}}(y-x)\right)\right\| \\
& +\left(1-\frac{1}{h_{\bar{n}}}\right)\|x-y\| \\
& =\|x-y\|-\frac{1-\theta}{h_{\bar{n}}}\|x-y\|+\frac{\theta+1}{h_{\bar{n}}}\left\|x+h_{\bar{n}}\left(u_{\bar{n}}-x\right)-y\right\| \\
\leq & \|x-y\| .
\end{aligned}
$$

So, (3.7) is satisfied and the conclusion follows from Corollary 3.3.

Example 3.5. Let $X=\mathbb{R}^{2}, K=[(0,0),(1,0)]$, and

$$
F(t, 0)=\left[\left(0, \frac{t}{4}\right),\left(0, \frac{2+t}{4}\right)\right] \cup\left[\left(0, \frac{2+t}{4}\right),\left(1, \frac{2+t}{4}\right)\right]
$$


Observe that $F$ is a contraction with constant $k=1 / 4$. For $t \in] 0,1]$,

$$
\begin{aligned}
& d((t, 0), F(t, 0))= \begin{cases}\frac{t \sqrt{17}}{4}, & \text { if } t \in\left[0, \frac{2}{\sqrt{17}-1}\right], \\
\frac{2+t}{4}, & \text { if } t \in\left[\frac{2}{\sqrt{17}-1}, 1\right],\end{cases} \\
& \{y \in F(t, 0):\|(t, 0)-y\|=d((t, 0), F(t, 0))\} \\
& = \begin{cases}\left\{\left(0, \frac{t}{4}\right)\right\} & \text { if } t \in\left[0, \frac{2}{\sqrt{17}-1}\right], \\
\left\{\left(0, \frac{t}{4}\right),\left(t, \frac{2+t}{4}\right)\right\} & \text { if } t=\frac{2}{\sqrt{17}-1}, \\
\left\{\left(t, \frac{2+t}{4}\right)\right\} & \text { if } \left.t \in] \frac{2}{\sqrt{17}-1}, 1\right] .\end{cases}
\end{aligned}
$$

Observe that for every $u \in K \backslash\{(1,0)\},\|u-(1,3 / 4)\|>3 / 4$. So (3.7) and hence (3.8) are not satisfied. Now, fix $\theta=1 / 2$. For $(t, 0) \in K$, choose $y=(0, t / 4) \in F(t, 0)$. Observe that $\|(t, 0)-y\|>d((t, 0), F(t, 0))$ if $t \in] 2 /(\sqrt{17}-1), 1]$. However, for every $t \in[0,1]$, choosing $u=(0,0)$, one has

$$
\theta\|(t, 0)-u\|+\|u-y\|=\frac{3}{4} t \leq d((t, 0), F(t, 0))
$$

Thus, Condition (3.1) is satisfied.

Observe that if $f: K \rightarrow X$ is a single-valued contraction satisfying (2.1) then for every $x \in K$, such that $x \neq f(x)$,

$$
f(x) \in\{y \in X: \exists u \in K \backslash\{x\} \text { such that } \theta d(x, u)+d(u, y) \leq d(x, y)\} .
$$

An analogous condition in the multivalued context leads to the following result.

Theorem 3.6. Let $K$ be a closed subset of $X$, and let $F: K \rightarrow X$ be a multivalued contraction with constant $k \in[0,1[$. Assume that there exits $\theta \in] k, 1]$ such that for every $x \in K, x \in F(x)$ or

$$
F(x) \in\{Y \subset X \text { nonempty and closed }: \exists u \in K \backslash\{x\} \text { such that } \theta d(x, u)+d(u, Y) \leq d(x, Y)\}
$$

Then F has a fixed point.

Proposition 3.7. Theorems 3.1 and 3.6 are equivalent.

Proof. It is clear that if (3.1) is satisfied, then (3.14) is also satisfied. Thus, Theorem 3.6 implies Theorem 3.1. 
Now, if assumptions of Theorem 3.6 are satisfied with some $\theta \in] k, 1]$. Fix $\varepsilon>0$ such that $\theta-\varepsilon>k$. Let $x \in K$. If $x \notin F(x)$, there exists $u \in K \backslash\{x\}$ such that

$$
\theta d(x, u)+d(u, F(x)) \leq d(x, F(x))
$$

Choose $y \in F(x)$ such that $d(u, y) \leq d(u, F(x))+\varepsilon d(x, u)$. So

$$
\widehat{\theta} d(x, u)+d(u, y) \leq d(x, F(x))
$$

where $\widehat{\theta}=\theta-\varepsilon$. Hence assumptions of Theorem 3.1 are satisfied with $\widehat{\theta}$.

As before, looking at the proof of Theorem 3.1, we see that we can relax the assumption that $F$ is a contraction.

Theorem 3.8. Let $K$ be a closed subset of $X$ and let $F: K \rightarrow X$ be a multivalued map with nonempty, closed values such that the map $x \mapsto d(x, F(x))$ is lower semicontinuous. Assume that there exist $k \in[0,1[$ and $\theta \in] k, 1]$ such that for every $x \in K$,

$$
\begin{gathered}
\emptyset \neq F(x) \cap(\{x\} \cup\{y \\
\in X: \exists u \in K \backslash\{x\} \text { such that } d(y, F(u)) \leq k d(x, u) \\
\leq \theta d(x, u)+d(u, y) \leq d(x, F(x))\}) .
\end{gathered}
$$

Then F has a fixed point.

We obtain as corollary a result due to Song [5] which generalizes a fixed point result due to Clarke [16].

Corollary 3.9 (Song [5]). Let $K$ be a closed nonempty subset of $X$, and let $F: K \rightarrow X$ be a multivalued with nonempty, closed, bounded values such that

(i) $F$ is H-upper semicontinuous, that is, for every $\varepsilon>0$ and every $x \in K$ there exists $r>0$ such that $\sup _{y \in F\left(x^{\prime}\right)} d(y, F(x))<\varepsilon$ for every $x^{\prime} \in B(x, r)$;

(ii) there exist $\alpha \in] 0,1]$, and $k \in[0, \alpha[$ such that for every $x \in K$ with $x \notin F(x)$, there exists $u \in K \backslash\{x\}$ satisfying

$$
\begin{gathered}
\alpha d(x, u)+d(u, F(x)) \leq d(x, F(x)), \\
\sup _{y \in F(x)} d(y, F(u)) \leq k d(x, u) .
\end{gathered}
$$

Then F has a fixed point.

Uderzo [6] generalized Song's result introducing the notion of directional multivalued $k(\cdot)$-contraction (this means that $F$ satisfies the following condition (ii)). This notion generalizes the notion of directional contractions used by Song [5] (Condition (ii) in Corollary 3.9). We show how Uderzo's result can be obtained from Theorem 3.8. 
Corollary 3.10 (Uderzo [6]). Let $K$ be a closed nonempty subset of $X$, and let $F: K \rightarrow X$ be a multivalued with nonempty, closed, bounded values such that

(i) $F$ is H-upper semicontinuous;

(ii) there exist $\alpha \in] 0,1], a:] 0, \infty[\rightarrow[\alpha, 1]$ and $k:] 0, \infty[\rightarrow[0,1[$ such that for every $x \in K$ with $x \notin F(x)$, there exists $u \in K \backslash\{x\}$ satisfying

$$
\begin{gathered}
a(d(x, u)) d(x, u)+d(u, F(x)) \leq d(x, F(x)), \\
\sup _{y \in F(x)} d(y, F(u)) \leq k(d(x, u)) d(x, u)
\end{gathered}
$$

(iii) there exist $x_{0} \in K$ and $\delta>0$ such that $d\left(x_{0}, F\left(x_{0}\right)\right) \leq \alpha \delta$;

(iv) $\sup _{t \in] 0, \delta]} k(t)<\inf _{t \in] 0, \delta]} a(t)$.

Then $F$ has a fixed point.

Proof. It is known that the $H$-upper semicontinuity of $F$ implies that $x \mapsto d(x, F(x))$ is lower semicontinuous. Let $\widehat{K}=\{x \in K: d(x, F(x)) \leq \alpha \delta\}$. This set is closed and nonempty. such that

Let $x \in \widehat{K}$ be such that $x \notin F(x)$. Assumption (ii) implies that there exists $u \in K \backslash\{x\}$

$$
\alpha d(x, u) \leq a(d(x, u)) d(x, u)+d(u, F(x)) \leq d(x, F(x)) \leq \alpha \delta .
$$

So $d(x, u) \leq \delta$. This inequality with (ii) and (iv) implies that

$$
\begin{aligned}
d(u, F(u)) & \leq d(u, F(x))+\sup _{y \in F(x)} d(y, F(u)) \\
& \leq d(x, F(x))-a(d(x, u)) d(x, u)+k(d(x, u)) d(x, u) \\
& \leq \alpha \delta .
\end{aligned}
$$

So $u \in \widehat{K} \backslash\{x\}$.

Denote

$$
\bar{k}=\sup _{t \in] 0, \delta]} k(t), \quad \bar{a}=\inf _{t \in] 0, \delta]} a(t) .
$$

Fix $\theta \in] \bar{k}, \bar{a}[$. Since $x \neq u$, choose $y \in F(x)$ such that

$$
d(u, y) \leq d(u, F(x))+(\bar{a}-\theta) d(x, u)
$$

So, by (ii),

$$
\begin{aligned}
d(y, F(u)) \leq \bar{k} d(x, u) \leq \theta d(x, u)+d(u, y) & \leq \bar{a} d(x, u)+d(u, F(x)) \\
& \leq d(x, F(x)) .
\end{aligned}
$$


So, the restriction $F: \widehat{K} \rightarrow X$ satisfies the assumptions of Theorem 3.8, and hence $F$ has a fixed point.

\section{Local Fixed Point Theorems for Generalized Inward Contractions}

In this section, we present local versions of fixed point theorems for generalized inward contractions.

Theorem 4.1. Let $K$ be a closed subset of $X, x_{0} \in K, r>0$, and let $F: B\left(x_{0}, r\right) \cap K \rightarrow X$ be a multivalued map with nonempty values and closed graph. Assume that there exist $c>0$ and $\tilde{d}$ an equivalent metric on $\mathrm{K} \times \mathrm{X}$ such that

(i) $c d\left(x_{1}, x_{2}\right) \leq \tilde{d}\left(\left(x_{1}, y_{1}\right),\left(x_{2}, y_{2}\right)\right)$ for every $x_{1}, x_{2} \in K$, and $y_{1}, y_{2} \in X$;

(ii) $d\left(x_{0}, F\left(x_{0}\right)\right)<c r$;

(iii) for every $x \in B\left(x_{0}, r\right) \cap K$ and every $y \in F(x) \backslash\{x\}$,

$\exists u \in K \backslash\{x\}, \quad \exists v \in X$ such that $\tilde{d}((x, y),(u, v))+d(u, v) \leq d(x, y)$, and

$$
v \in F(u) \text { if } u \in B\left(x_{0}, r\right) \text {. }
$$

Then F has a fixed point.

Proof. Choose $\tilde{r} \in] 0, r\left[\right.$ and such that $d\left(x_{0}, F\left(x_{0}\right)\right)<c \tilde{r}$. Fix $y_{0} \in F\left(x_{0}\right)$ such that

$$
d\left(x_{0}, y_{0}\right)<c \tilde{r}
$$

Consider

$$
Y=\left\{(x, y) \in\left(\overline{B\left(x_{0}, \tilde{r}\right)} \times X\right) \cap \text { graph } F: \tilde{d}\left(\left(x_{0}, y_{0}\right),(x, y)\right)+d(x, y) \leq d\left(x_{0}, y_{0}\right)\right\}
$$

This space endowed with the metric $\tilde{d}$ is a nonempty complete metric space since $\left(x_{0}, y_{0}\right) \in Y$.

Applying the Bisholp-Phelps Theorem (Theorem 1.4) insures the existence of $\left(x^{*}, y^{*}\right) \in$ $Y$ satisfying

$$
\tilde{d}\left((x, y),\left(x^{*}, y^{*}\right)\right)+d(x, y)>d\left(x^{*}, y^{*}\right) \quad \forall(x, y) \in Y \backslash\left\{\left(x^{*}, y^{*}\right)\right\}
$$

If $y^{*}=x^{*}$ then $x^{*}$ is a fixed point of $F$.

If not, by assumption (iii), there exists $x \in K \backslash\left\{x^{*}\right\}$ and $y \in X$ such that

$$
\tilde{d}\left((x, y),\left(x^{*}, y^{*}\right)\right)+d(x, y) \leq d\left(x^{*}, y^{*}\right) .
$$


This inequality combined with the fact that $\left(x^{*}, y^{*}\right) \in Y$ implies that

$$
\begin{aligned}
\tilde{d}\left(\left(x_{0}, y_{0}\right),(x, y)\right) & \leq \tilde{d}\left(\left(x_{0}, y_{0}\right),\left(x^{*}, y^{*}\right)\right)+\tilde{d}\left((x, y),\left(x^{*}, y^{*}\right)\right) \\
& \leq d\left(x_{0}, y_{0}\right)-d\left(x^{*}, y^{*}\right)+d\left(x^{*}, y^{*}\right)-d(x, y) \\
& =d\left(x_{0}, y_{0}\right)-d(x, y) .
\end{aligned}
$$

From (4.2), (4.6) and Assumption (i) we deduce

$$
c d\left(x, x_{0}\right) \leq \tilde{d}\left(\left(x_{0}, y_{0}\right),(x, y)\right) \leq d\left(x_{0}, y_{0}\right)-d(x, y) \leq c \tilde{r}
$$

So, $x \in \overline{B\left(x_{0}, \widetilde{r}\right)} \cap K$, and by (iii), $y \in F(x)$. Thus, by (4.6), $(x, y) \in Y$.

Therefore, (4.5) contracdicts (4.4). So, $F$ has a fixed point.

Corollary 4.2. Let $K$ be a closed subset of $X, x_{0} \in K, r>0$, and let $F: B\left(x_{0}, r\right) \cap K \rightarrow X$ be a multivalued map with nonempty values and closed graph. Assume that there exist $\alpha, \beta>0$ such that

(i) $d\left(x_{0}, F\left(x_{0}\right)\right)<\alpha r$;

(ii) for every $x \in B\left(x_{0}, r\right) \cap K$ and every $y \in F(x) \backslash\{x\}$,

$\exists u \in K \backslash\{x\}, \quad \exists v \in X$ such that $\alpha d(x, u)+d(u, v)+\beta d(v, y) \leq d(x, y)$, and

$$
v \in F(u) \text { if } u \in B\left(x_{0}, r\right) \text {. }
$$

Then F has a fixed point.

As corollaries, we obtain local versions of Theorems 2.1 and 2.3.

Theorem 4.3. Let $K$ be a closed subset of $X, x_{0} \in K, r>0$, and let $F: B\left(x_{0}, r\right) \cap K \rightarrow X$ be a multivalued map with nonempty values and closed graph. Assume that there are constants $k \in[0,1[$ and $\theta \in] k, 1]$ such that

(i) $d\left(x_{0}, F\left(x_{0}\right)\right)<(\theta-k) r$;

(ii) for all $x \in B\left(x_{0}, r\right) \cap K$,

$$
\begin{gathered}
F(x) \subset\{x\} \cup\{y \in X: \exists u \in K \backslash\{x\} \text { such that } \theta d(x, u)+d(u, y) \leq d(x, y) \text {, and if } \\
\left.u \in B\left(x_{0}, r\right), d(y, F(u)) \leq k d(x, u)\right\} .
\end{gathered}
$$

Then F has a fixed point.

Proof. Choose $\tilde{k}>k$ such that $d\left(x_{0}, F\left(x_{0}\right)\right)<(\theta-\tilde{k}) r$. Let $\left.\delta \in\right] 0,(\tilde{k} / k)-1[$. Consider on $K \times X$ the metric

$$
\tilde{d}\left(\left(x_{1}, y_{1}\right),\left(x_{2}, y_{2}\right)\right)=(\theta-\tilde{k}) d\left(x_{1}, x_{2}\right)+\delta d\left(y_{1}, y_{2}\right)
$$


The conclusion follows from Theorem 4.1 if we show that Condition (iii) holds. Let $x \in$ $B\left(x_{0}, r\right) \cap K$ and $y \in F(x) \backslash\{x\}$, and let $u \in K \backslash\{x\}$ be given by Assumption (ii). If $u \notin B\left(x_{0}, r\right)$, choose $v=y$, and if $\mathrm{u} \in B\left(x_{0}, r\right)$, choose $v \in F(u)$ such that $d(y, v) \leq \widetilde{k} d(x, u) /(1+\delta)$. So,

$$
\begin{aligned}
\tilde{d}((x, y),(u, v))+d(u, v) & \leq(\theta-\tilde{k}) d(x, u)+\delta d(y, v)+d(u, y)+d(y, v) \\
& \leq \theta d(x, u)+d(u, y) \\
& \leq d(x, y) .
\end{aligned}
$$

In the case where $F$ is a contraction, the previous result can be stated more simply.

Corollary 4.4. Let $K$ be a closed subset of $X, x_{0} \in K$, and $r>0$. Assume that $F: B\left(x_{0}, r\right) \cap K \rightarrow X$ is a multivalued contraction with constant $k \in[0,1[$ for which there exits $\theta \in] k, 1]$ such that

(i) $d\left(x_{0}, F\left(x_{0}\right)\right)<(\theta-k) r$;

(ii) $F(x) \subset\{x\} \cup\{y \in X: \exists u \in K \backslash\{x\}$ such that $\theta d(x, u)+d(u, y) \leq d(x, y)\}$ for all $x \in B\left(x_{0}, r\right) \cap K$.

Then F has a fixed point.

We obtain as corollary the following result due to Maciejewski [4].

Corollary 4.5. Let $K$ be a closed subset of $X, x_{0} \in K$, and $r>0$. Assume that $F: B\left(x_{0}, r\right) \cap K \rightarrow X$ is a multivalued contraction with constant $k \in[0,1$ [ such that

(i) $d\left(x_{0}, F\left(x_{0}\right)\right)<(1-k) r$;

(ii) $F(x) \subset \widetilde{I}_{K}(x)$ for all $x \in B\left(x_{0}, r\right) \cap K$, where $\widetilde{I}_{K}(x)$ is defined in (2.8).

Then $F$ has a fixed point.

Proof. Choose $\delta \in] 0,(1-k) /(1+k)[$ and $\theta \in] k,(1-\delta)(1+\delta)\left[\right.$ be such that $d\left(x_{0}, F\left(x_{0}\right)\right)<$ $(\theta-k) r$. Arguing as in the proof of Corollary 2.2, one sees that Assumption (ii) implies that for every $x \in B\left(x_{0}, r\right) \cap K$,

$$
F(x) \subset\{x\} \cup\{y \in X: \exists u \in K \backslash\{x\} \text { such that } \theta d(x, u)+d(u, y) \leq d(x, y)\} .
$$

The conclusion follows from Corollary 4.4.

Fixed point results can also be obtained for multivalued maps defined on a ball of $K$ and satisfying an intersection condition. Here is a local version of Theorem 3.8.

Theorem 4.6. Let $K$ be a closed subset of $X, x_{0} \in K, r>0$, and let $F: B\left(x_{0}, r\right) \cap K \rightarrow X$ be a multivalued map with nonempty, closed values such that $x \mapsto d(x, F(x))$ is lower semicontinuous. 
Assume that there exist $k \in[0,1[$ and $\theta \in] k, 1]$ such that

(i) $d\left(x_{0}, F\left(x_{0}\right)\right)<(\theta-k) r$;

(ii) for every $x \in B\left(x_{0}, r\right) \cap K$,

$$
\begin{gathered}
\emptyset \neq F(x) \cap(\{x\} \cup\{y \in X: \exists u \in K \backslash\{x\} \\
\text { such that } \theta d(x, u)+d(u, y) \leq d(x, F(x)) \text { and if } \\
\left.\left.\qquad u \in B\left(x_{0}, r\right), d(y, F(u)) \leq k d(x, u)\right\}\right) .
\end{gathered}
$$

Then F has a fixed point.

Proof. Choose $\tilde{r} \in] 0, r\left[\right.$ such that $d\left(x_{0}, F\left(x_{0}\right)\right)<(\theta-k) \tilde{r}$. Consider

$$
Z=\left\{x \in \overline{B\left(x_{0}, \tilde{r}\right)} \cap K:(\theta-k) d\left(x_{0}, x\right)+d(x, F(x)) \leq d\left(x_{0}, F\left(x_{0}\right)\right)\right\} .
$$

The space $Z$ is a nonempty closed subset of $X$ since $x_{0} \in Z$ and $x \mapsto d(x, F(x))$ is lower semicontinuous.

Applying the Bisholp-Phelps Theorem to $\phi(x)=d(x, F(x))$ insures the existence of $x^{*} \in Z$ such that

$$
(\theta-k) d\left(x, x^{*}\right)>d\left(x^{*}, F\left(x^{*}\right)\right)-d(x, F(x)) \quad \forall x \in Z \backslash\left\{x^{*}\right\} .
$$
such that

If $x^{*}$ is not a fixed point of $F$, by Assumption (ii), there exist $y^{*} \in F\left(x^{*}\right)$ and $x \in K \backslash\left\{x^{*}\right\}$

$$
\begin{gathered}
\theta d\left(x^{*}, x\right)+d\left(x, y^{*}\right) \leq d\left(x^{*}, F\left(x^{*}\right)\right), \text { and } \\
d\left(y^{*}, F(x)\right) \leq k d\left(x, x^{*}\right) \quad \text { if } x \in B\left(x_{0}, r\right) .
\end{gathered}
$$

By Assumption (i) and since $x^{*} \in Z$,

$$
\begin{aligned}
(\theta-k) d\left(x, x_{0}\right) & \leq(\theta-k) d\left(x^{*}, x_{0}\right)+(\theta-k) d\left(x, x^{*}\right) \\
& \leq d\left(x_{0}, F\left(x_{0}\right)\right)-d\left(x^{*}, F\left(x^{*}\right)\right)+d\left(x^{*}, F\left(x^{*}\right)\right)-d\left(x, y^{*}\right)-k d\left(x^{*}, x\right) \\
& \leq d\left(x_{0}, F\left(x_{0}\right)\right) \leq(\theta-k) \tilde{r} .
\end{aligned}
$$

So, $x \in \overline{B\left(x_{0}, \widetilde{r}\right)} \cap K \subset B\left(x_{0}, r\right)$. From (4.16), we deduce

$$
\begin{aligned}
(\theta-k) d\left(x, x_{0}\right)+d(x, F(x)) \leq & (\theta-k) d\left(x_{0}, x^{*}\right)+(\theta-k) d\left(x^{*}, x\right) \\
& +d\left(x, y^{*}\right)+d\left(y^{*}, F(x)\right) \\
\leq & (\theta-k) d\left(x_{0}, x^{*}\right)+d\left(x^{*}, F\left(x^{*}\right)\right) \\
\leq & d\left(x_{0}, F\left(x_{0}\right)\right) .
\end{aligned}
$$

Hence, $x \in Z$. 
By (4.16),

$$
\begin{aligned}
(\theta-k) d\left(x, x^{*}\right)+d(x, F(x)) & \leq(\theta-k) d\left(x, x^{*}\right)+d\left(x, y^{*}\right)+d\left(y^{*}, F(x)\right) \\
& \leq d\left(x^{*}, F\left(x^{*}\right)\right)
\end{aligned}
$$

this contradicts (4.15) since $x \neq x^{*}$. So $F$ has a fixed point.

\section{Continuation Principle for Generalized Inward Contractions}

In this section, we obtain continuation principles for families of contractions satisfying a generalized inwardness condition. For $K \subset X$ and $U$ open in $K$, we denote $\partial_{K} U$ the boundary of $U$ relative to $K$. Here is a generalization of Theorem 4.3 in [17]. The proof is analogous.

Theorem 5.1. Let $K$ be a closed subset of $X, U$ open in $K, k \in[0,1[, \theta \in] k, 1]$, and $\phi:[0,1] \rightarrow \mathbb{R}$ continuous and increasing. Assume $H: \bar{U} \times[0,1] \rightarrow X$ is a multivalued map with nonempty values and closed graph such that

(i) $x \notin H(x, t) \forall x \in \partial_{K} U$ and for all $t \in[0,1]$;

(ii) $D(H(x, t), H(x, s))<|\phi(t)-\phi(s)|$ for all $x \in \bar{U}$ and all $t, s \in[0,1]$;

(iii) for all $x \in \bar{U}$, and all $t \in[0,1]$,

$$
\begin{gathered}
H(x, t) \subset\{x\} \cup\{y \in X: \exists u \in K \backslash\{x\} \text { such that } \theta d(x, u)+d(u, y) \leq d(x, y), \\
\text { and if } u \in \bar{U}, d(y, H(u, t)) \leq k d(x, u)\}
\end{gathered}
$$

Then $H(\cdot, 0)$ has a fixed point if and only if $H(\cdot, 1)$ has a fixed point.

Proof. Consider

$$
Q=\{(x, t) \in \bar{U} \times[0,1]: x \in H(x, t)\},
$$

endowed with the partial order

$$
(x, s) \leq(y, t) \Longleftrightarrow s \leq t, \quad d(x, y) \leq \frac{2(\phi(t)-\phi(s))}{\theta-k} .
$$

Assuming that $H(\cdot, 0)$ has a fixed point implies that $Q$ is nonempty. It is easy to show that every totally ordered subset of $Q$ has a an upper bound. Hence, by Zorn's Lemma, $Q$ has a maximal element $\left(x_{0}, t_{0}\right)$. By (i), $x_{0} \in U$. To conclude we need to show that $t_{0}=1$. If not, there is $r>0$ such that $B\left(x_{0}, r\right) \cap K \subset U$, and there exist $\left.\left.\widehat{t} \in\right] t_{0}, 1\right]$ and $\left.r_{0} \in\right] 0, r$ [ such that $2\left(\phi(\widehat{t})-\phi\left(t_{0}\right)\right)=(\theta-k) r_{0}$. Therefore,

$$
d\left(x_{0}, H\left(x_{0}, \hat{t}\right)\right) \leq d\left(x_{0}, H\left(x_{0}, t_{0}\right)\right)+D\left(H\left(x_{0}, t_{0}\right), H\left(x_{0}, \hat{t}\right)\right) \leq \phi(\hat{t})-\phi\left(t_{0}\right)<(\theta-k) r_{0} .
$$


Thus, $H(\cdot, \widehat{t})$ satisfies the assumptions of Theorem 4.3 , and hence has a fixed point $\widehat{x}$. This contradicts the maximality of $\left(x_{0}, t_{0}\right)$ since $\left(x_{0}, t_{0}\right)<(\widehat{x}, \widehat{t})$.

Corollary 5.2. Let $E$ be a uniformly convex Banach space, $U \subset E$ open, bounded, convex, and $K \subset E$ closed, convex such that $0 \in U \cap K$. Assume that $f: \bar{U} \cap K \rightarrow E$ is a nonexpansive map such that

(i) $x \neq \lambda f(x) \forall x \in \partial_{K} U$ and for all $\left.\lambda \in\right] 0,1$ [;

(ii) there exists a lower semicontinuous map $\lambda \mapsto \theta_{\lambda}$ defined on $\left[0,1\left[\right.\right.$ with $\theta_{\lambda}>\lambda$ and such that for every $x \in \bar{U}, x=\lambda f(x)$ or there exists $u \in K \backslash\{x\}$ such that $\theta_{\lambda}\|x-u\|+\|u-\lambda f(x)\| \leq$ $\|x-\lambda f(x)\|$.

Then $f$ has a fixed point.

Proof. Observe that for $\lambda_{1}, \lambda_{2} \in[0,1]$,

$$
\begin{aligned}
\left\|\lambda_{1} f(x)-\lambda_{2} f(x)\right\| & \leq\left|\lambda_{1}-\lambda_{2}\right|(\|f(x)-f(0)\|+\|f(0)\|) \\
& \leq\left|\lambda_{1}-\lambda_{2}\right|(\|x\|+\|f(0)\|) \\
& \leq M\left|\lambda_{1}-\lambda_{2}\right|
\end{aligned}
$$

for every $x \in \bar{U} \cap K$ since $\bar{U}$ is bounded.

Assumption (ii) implies that for every $\lambda \in\left[0,1\left[\right.\right.$, there exists $\left.\delta_{\lambda} \in\right] 0,\left(\theta_{\lambda}-\lambda\right) / 4[$ such that for every $\gamma \in] \lambda-2 \delta_{\lambda}, \lambda+2 \delta_{\lambda}[\cap[0,1[$,

$$
\theta_{\gamma} \geq \widehat{\theta}_{\lambda}>\lambda+2 \delta_{\lambda}
$$

where $\widehat{\theta}_{\lambda}=\theta_{\lambda}-\left(\theta_{\lambda}-\lambda\right) / 4$. So, \{]$\lambda-\delta_{\lambda}, \lambda+\delta_{\lambda}[: \lambda \in[0,1[\}$ is an open cover of $[0,1[$. Thus, there exists an increasing sequence $\left\{\lambda_{n}\right\}$ in $\left[0,1\right.$ [ converging to 1 such that \{]$\lambda_{n}-\delta_{\lambda_{n}}, \lambda_{n}+\delta_{\lambda_{n}}[: n \in$ $\mathbb{N}\}$ is an open cover of $\left[0,1\left[\right.\right.$. Denote $\lambda_{n}^{-}=\lambda_{n}-\delta_{\lambda_{n}}, \lambda_{n}^{+}=\lambda_{n}+\delta_{\lambda_{n}}$ and $\bar{\theta}_{n}=\hat{\theta}_{\lambda_{n}}$. By construction and by Assumption (ii), for every $n \in \mathbb{N}$ and every $\lambda \in\left[\lambda_{n}^{-}, \lambda_{n}^{+}\right]$, we have that for every $x \in \bar{U}$ such that $x \neq \lambda f(x)$, there exists $u \in K \backslash\{x\}$ such that

$$
\bar{\theta}_{n}\|x-u\|+\|u-\lambda f(x)\| \leq\|x-\lambda f(x)\|
$$

The previous theorem applied inductively to $H_{n}(x, t)=\left((1-t) \lambda_{n}^{-}+t \lambda_{n}^{+}\right) f(x)$ insures the existence of $x_{n} \in U \cap K$ such that $x_{n}=\lambda_{n}^{+} f\left(x_{n}\right)$. The sequence $\left\{x_{n}\right\}$ has a weakly converging subsequence still denoted $\left\{x_{n}\right\}$ such that $x_{n}-f\left(x_{n}\right) \rightarrow 0$. The demi-closedness of $I-f$ (see $[18$, Theorem 3]) implies that $f$ has a fixed point.

Similarly to Theorem 5.1, we can prove the following continuation principles using Theorems 4.1 and 4.6 , respectively.

Theorem 5.3. Let $K$ be a closed subset of $X, U$ open in $K, \phi:[0,1] \rightarrow \mathbb{R}$ continuous and increasing, and $H: \bar{U} \times[0,1] \rightarrow X$ a multivalued map with nonempty values and closed graph. Assume that 
there exist $c>0$ and $\tilde{d}$ an equivalent metric on $K \times X$ such that

(i) $x \notin H(x, t) \forall x \in \partial_{K} U$ and for all $t \in[0,1]$;

(ii) $D(H(x, t), H(x, s))<|\phi(t)-\phi(s)|$ for all $x \in \bar{U}$ and all $t, s \in[0,1]$;

(iii) $c d\left(x_{1}, x_{2}\right) \leq \tilde{d}\left(\left(x_{1}, y_{1}\right),\left(x_{2}, y_{2}\right)\right)$ for every $x_{1}, x_{2} \in K$, and $y_{1}, y_{2} \in X$;

(iv) for every $(x, t) \in \bar{U} \times[0,1]$ and every $y \in H(x, t) \backslash\{x\}$,

$\exists u \in K \backslash\{x\}, \quad \exists v \in X$ such that $\tilde{d}((x, y),(u, v))+d(u, v) \leq d(x, y)$ and

$$
v \in H(u, t) \text { if } u \in \bar{U} \text {. }
$$

Then $H(\cdot, 0)$ has a fixed point if and only if $H(\cdot, 1)$ has a fixed point.

Theorem 5.4. Let $K$ be a closed subset of $X, U$ open in $K, k \in[0,1[, \theta \in] k, 1]$ and and $\phi:[0,1] \rightarrow$ $\mathbb{R}$ continuous and increasing. Assume $H: \bar{U} \times[0,1] \rightarrow X$ is a multivalued with nonempty, closed values such that

(i) $x \notin H(x, t)$ for all $x \in \partial_{K} U$ and all $t \in[0,1]$;

(ii) $D(H(x, t), H(x, s))<|\phi(t)-\phi(s)|$ for all $x \in \bar{U}$ and all $t, s \in[0,1]$;

(iii) $x \mapsto d(x, H(x, t))$ is lower semicontinuous for all $t \in[0,1]$;

(iv) for all $x \in \bar{U}$, and all $t \in[0,1]$,

$$
\begin{gathered}
\emptyset \neq H(x, t) \cap\{x\} \cup\{y \in X: \exists u \in K \backslash\{x\} \\
\text { such that } \theta d(x, u)+d(u, y) \leq d(x, H(x, t)) \text { and if } \\
u \in \bar{U}, d(y, H(u, t)) \leq k d(x, u)\} .
\end{gathered}
$$

Then $H(\cdot, 0)$ has a fixed point if and only if $H(\cdot, 1)$ has a fixed point.

\section{Acknowledgment}

This work was partially supported by CRSNG Canada.

\section{References}

[1] S. B. Nadler Jr., "Multivalued contraction mappings," Pacific Journal of Mathematics, vol. 30, no. 2, pp. 475-487, 1969.

[2] T.-C Lim, "A fixed point theorem for weakly inward multivalued contractions," Journal of Mathematical Analysis and Applications, vol. 247, no. 1, pp. 323-327, 2000.

[3] D. Azé and J.-N. Corvellec, "A variational method in fixed point results with inwardness conditions," Proceedings of the American Mathematical Society, vol. 134, no. 12, pp. 3577-3583, 2006.

[4] M. Maciejewski, "Inward contractions on metric spaces," Journal of Mathematical Analysis and Applications, vol. 330, no. 2, pp. 1207-1219, 2007.

[5] W. Song, "A generalization of Clarke's fixed point theorem," Applied Mathematics: A Journal of Chinese Universities. Series B, vol. 10, no. 4, pp. 463-466, 1995. 
[6] A. Uderzo, "Fixed points for directional multi-valued $k(\cdot)$-contractions," Journal of Global Optimization, vol. 31, no. 3, pp. 455-469, 2005.

[7] J. Caristi, "Fixed point theorems for mappings satisfying inwardness conditions," Transactions of the American Mathematical Society, vol. 215, pp. 241-251, 1976.

[8] I. Ekeland, "Sur les problèmes variationnels," Comptes Rendus de l'Académie des Sciences, Série A-B, vol. 275, pp. 1057-1059, 1972.

[9] I. Ekeland, "Nonconvex minimization problems," Bulletin of the American Mathematical Society, vol. 1, no. 3, pp. 443-474, 1979.

[10] J. Dugundji and A. Granas, Fixed Point Theory. I, vol. 61 of Mathematical Monographs, Polish Scientific Publishers, Warszawa, Poland, 1982.

[11] A. Granas and J. Dugundji, Fixed Point Theory, Springer Monographs in Mathematics, Springer, New York, NY, USA, 2003.

[12] E. Bishop and R. R. Phelps, "The support functionals of a convex set," in Proc. Sympos. Pure Math., Vol. VII, V. Klee, Ed., American Mathematical Society, Providence, RI, USA, 1963.

[13] R. R. Phelps, "Support cones in Banach spaces and their applications," Advances in Mathematics, vol. 13, pp. 1-19, 1974.

[14] N. Mizoguchi and W. Takahashi, "Fixed point theorems for multivalued mappings on complete metric spaces," Journal of Mathematical Analysis and Applications, vol. 141, no. 1, pp. 177-188, 1989.

[15] D. Downing and W. A. Kirk, "Fixed point theorems for set-valued mappings in metric and Banach spaces," Mathematica Japonica, vol. 22, no. 1, pp. 99-112, 1977.

[16] F. Clarke, "Pointwise contraction criteria for the existence of fixed points," Canadian Mathematical Bulletin, vol. 21, no. 1, pp. 7-11, 1978.

[17] M. Frigon and A. Granas, "Résultats du type de Leray-Schauder pour des contractions multivoques," Topological Methods in Nonlinear Analysis, vol. 4, no. 1, pp. 197-208, 1994.

[18] F. E. Browder, "Semicontractive and semiaccretive nonlinear mappings in Banach spaces," Bulletin of the American Mathematical Society, vol. 74, pp. 660-665, 1968. 